\title{
Application of Laplace Decomposition Method to Solve Linear and Nonlinear Heat Equation
}

\author{
Sujit Handibag and B. D. Karande
}

\begin{abstract}
In this paper, we develop a method to obtain approximate solution of nonlinear heat equation with the help of Laplace Decomposition Method (LDM). The technique is based on the application of Laplace transform to nonlinear partial differential equation. The nonlinear term can easily be handle with the help of Adomian polynomials. We illustrate this technique with the help of three examples and results of the present technique have closed arrestment with approximate solutions obtained with the help of Adomian Decomposition Method (ADM).
\end{abstract}

Index Terms-Approximate solution, Laplace decomposition method, nonlinear partial differential equations. Adomian decomposition method.

\section{INTRODUCTION}

The Adomian decomposition method (ADM) introduced by Adomian possesses a great potential in solving different kind of functional equation. The method is very well suited to physical problems since it does not require unnecessary liberalization, perturbation and other restrictive methods and assumptions which may change the problem begin solved sometime seriously.

The Laplace Decomposition Method (LDM) is a numerical algorithm to solve nonlin- ear ordinary, partial differential equation. Khuri used this method for the approximate so- lution of a class of nonlinear ordinary differential equation. Agadjanov applied this method for the solution of Duffng equation. Elgazery exploit this method to solve Falkner-Skan equation. This numerical technique basically illustrates how the Laplace transform may be used to approximate the solutions by manipulating the decomposition method which was first introduction by Adomian. The present paper aims at offering an alternative method of solution to the existing ones concerning to the linear and nonlinear heat equation. By using Laplace transform base on decomposition method for solving heat equation

$$
u_{t}(x, t)=u_{x x}+\in u^{m}
$$

where $\mathrm{m}=1,2,3, \ldots \ldots \ldots \ldots . .$. and $\varepsilon$ is parameter, and the indices $t$ and $x$ denote derivatives with respect to these variables. Unless $\mathrm{m}=1$, equation (1.1) is a nonlinear heat equation.

Construction of particular exact solution for nonlinear equations of the form (1.1) is an important problem. Especially, finding an exact solution that has a biological

Manuscript received May 6, 2012; revised June 15, 2012.

S. Handibag is with department of Mathematics, Mahatma Basweshwar Mahavidyalaya, Latur-413 512, Maharashtra, India (tel.: +919011491162, e-mail: sujitmaths@gmail.com).

B. D. Karande is with Department of Mathematics, Maharashtra Udaygiri Mahavidyalaya, Udgir, India inter- pretation is of fundamental importance. In contrast to simple diffusion ( $\epsilon=0$ case), when reaction kinetics and diffusion are coupled, traveling waves of chemical concentration exist, can effect a biological change, very fast than straight diffusion processes governed by equa-tion like (1.1) with $\in=0$. This coupling gives rise to reaction diffusion equation of the form (1.1), where $u$ is concentration and the term $\in u_{m}$ represent the kinetics. For example, it is know that for $\mathrm{m}=3$, equation with cubic nonlinear that admits soliton like solutions.

In the following section we apply the Laplace decomposition method (LDM) to equation (1.1) to obtain the particular exact solution of it in $\mathrm{x}$ as well as $\mathrm{t}$ direction by taking

Laplace transform with respect to $\mathrm{x}$ and $\mathrm{t}$ respectively.

\section{LAPLACE DeCOMPOSITION METHOD}

The aim of this section is to discuss the use of Laplace transform algorithm for the nonlinear heat equations. We consider the general form of homogeneous nonlinear heat equations with initial conditions is given below

$$
\begin{gathered}
L u(x, t)+R u(x, t)+\epsilon u_{m}=0 \\
u(x, 0)=f(x), u(0, t)=g(t), u x(0, t)=h(t)
\end{gathered}
$$

where $L=\frac{\partial}{\partial x}, R=\frac{\partial^{2}}{\partial x^{2}}$ and $N u$ represent the general nonlinear operator $\in \mathrm{u}_{\mathrm{m}}$. Taking the

Laplace transform on both sides of eq. (2.2) with respect to $t$, we get

$$
L t[L u(x, t)]+\operatorname{Lt}[R u(x, t)]+\operatorname{Lt}[N u(x, t)]=0
$$

where $L_{t}$ represent the Laplace transform with respect to $t$.

$$
\begin{gathered}
s u(x, s)-u(x, 0)-\frac{1}{s} L_{t}\left[u_{x x}(x, t)+\epsilon u^{m}=0\right. \\
u(x, s)=\frac{1}{s} f(x)+\frac{1}{s} L_{t}\left[u_{x x}(x, t)+\epsilon u^{m}\right]
\end{gathered}
$$

Taking inverse Laplace transform on both sides of equations (2.4) with respect to $t$, we get

$$
u(x, t)=f(x)+L_{t}^{-1}\left[\frac{1}{s} L_{t}\left[u_{x x}(x, t)+\epsilon u^{m}\right]\right]
$$

In Laplace Decomposition Method we represent solution in infinite series form. Therefore suppose that

$$
u(x, t)=\sum_{n=0}^{\infty} u_{n}(x, t)
$$


is a required solution of equation (2.2). A nonlinear term occurs eq. (2.2), we can decompose it by using Adomian polynomial, which is given by the formula

$$
A_{n}=\frac{1}{n !} \frac{d^{n}}{d \gamma^{n}}\left[N\left(\sum_{n=0}^{\infty} \gamma^{n} u_{n}\right)\right]_{\gamma=0}, \quad n \geq 0
$$

Therefore

$$
N u(x, t)=\sum_{m=0}^{\infty} A_{m}
$$

where $A_{n}$ are Adomian polynomials of $u_{0}, u_{1}, u_{2} \ldots \ldots u_{n}, n \geq$ 0 . Which are calculated by using eq. (2.7) We obtain the first few Adomian polynomials for as , $N u(x, t)=\epsilon u^{m}$ as

$$
\begin{aligned}
& A_{0}=\epsilon u_{0}^{m}, \quad A_{1}=m \epsilon u^{m-1}, \\
& A_{2}=\frac{m \epsilon}{2}\left[(m-1) u_{0}^{m-2} u_{1}^{2}+2 u_{2} u_{0}^{m-1}\right]
\end{aligned}
$$

and so on. Putting equations (2.6) and (2.8) in equation (2.5), we get

$$
\begin{gathered}
\sum_{n=0}^{\infty} u_{n}(x, t)=f(x)+L_{t}^{-1}\left[\frac { 1 } { s } L _ { t } \left[\sum_{n=0}^{\infty} u_{n x x}(x, t)\right.\right. \\
\left.\left.+\sum_{m=0}^{\infty} A_{m}\right]\right]
\end{gathered}
$$

Comparing the both sides of above equation, we get

$$
\begin{aligned}
u_{0}(x, t)=f(x), & u_{1}(x, t) \\
& =L_{t}^{-1}\left(\frac { 1 } { s } L _ { t } \left[u_{0 x x}(x, t)\right.\right. \\
& \left.\left.+A_{1}\right]\right), \quad u_{2}(x, t) \\
& =L_{t}^{-1}\left(\frac{1}{s} L_{t}\left[u_{1 x x}+A_{1}\right]\right)
\end{aligned}
$$

In general, the recursive relation is given by

$$
\begin{gathered}
u_{0}(x, t)=f(x), \quad u_{n+1}(x, t) \\
=L_{t}^{-1}\left(\frac{1}{s} L_{t}\left[u_{n x x}(x, t)+A_{n}\right]\right), \\
n \geq 0
\end{gathered}
$$

By using the above recursive relation, we can find the few components of $u(x, t)$; namely $u_{0}, u_{1}, u 2 \ldots \ldots, u_{n}, n \geq 0$. Substitute all these values in equation (2.6), we get the solution of eq. (2.2) in series form in t direction. When we take Laplace transform of (2.2) with respect to $\mathrm{x}$, we get the same solution in $\mathrm{x}$ direction. The recursive relation in $\mathrm{x}$ direction is,

$$
\begin{gathered}
u_{0}(x, t)=g(t)+x h(t), \\
u_{n+1}(x, t) \\
=L_{x}^{-1}\left(\frac{1}{s} L_{x}\left[u_{n t}(x, t)-A_{n}\right]\right), \\
n \geq 0
\end{gathered}
$$

To illustrate this method for coupled linear and nonlinear heat equation we take two examples in the following section.

\section{APPLICATIONS}

Example 1: If we take $\epsilon=1$ and $\mathrm{m}=1$ in equation (1.1), we obtain the linear heat equation, namely

$$
u_{t}=u_{x x}+u
$$

with initial condition $\mathrm{u}(\mathrm{x}, 0)=\cos (\pi \mathrm{x})$ and boundary conditions $u(0, t)=e^{\left(1-\pi^{2}\right) t}, u_{x}(0, t)=0$

Let us find the solution of equation (3.12) in $t$ direction, we use the recursive relation

(2. 10), we get

$$
\begin{gathered}
u_{0}(x, t)=\cos (\pi x) \\
u_{1}(x, t)=L_{t}^{-1}\left[\frac{1}{s} L_{t}\left[u_{0 x x}+A_{0}\right]\right] \\
=L_{t}^{-1}\left[\frac { 1 } { s } L _ { t } \left[-\pi^{2} \cos (\pi x)\right.\right. \\
\left.\left.+u_{0}\right]\right] \\
=L_{t}^{-1}\left[\frac{1}{s^{2}}\left[\left(1-\pi^{2}\right) \cos (\pi x)\right]\right]=t\left(1-\pi^{2}\right) \cos (\pi x) \\
u_{2}(x, t)=L_{t}^{-1}\left[\frac{1}{s} L_{t}\left[u_{1 x x}+A_{1}\right]\right]= \\
L_{t}^{-1}\left[\frac{1}{s} L_{t}\left[-t\left(1-\pi^{2}\right) \cos (\pi x)+t\left(1-\pi^{2}\right) \cos (\pi x)\right]\right] \\
=\frac{t^{2}}{2 !}\left(1-\pi^{2}\right) \cos (\pi x)
\end{gathered}
$$

and so on. In this manner the rest of components of the series (2.6) have been calculate using mathcad7. Putting these individual terms in (2.6) one we get the exact solution in $\mathrm{t}$

$$
\begin{aligned}
& \text { direction } \\
& \begin{array}{c}
u(x, t)=\cos (\pi x)+\left(1-\pi^{2}\right) t \cos (\pi x)+\frac{1}{2 !}(1- \\
\left.\pi^{2}\right) t^{2} \cos (\pi x)+\frac{1}{3 !}\left(1-\pi^{2}\right) t^{3} \cos (\pi x)+\cdots \\
=e^{\left(1-\pi^{2}\right) t} \cos (\pi x)
\end{array}
\end{aligned}
$$

which can be verified through substitution. Similarly, Let us find the solution in $\mathrm{x}$ direction, we use the recursive relation (2.11), we get

$$
\begin{aligned}
& u_{0}(x, t)=e^{\left(1-\pi^{2}\right) t} \\
& u_{1}(x, t)=L_{x}^{-1}\left[\frac{1}{s^{2}} L_{x}\left[u_{0 t}+A_{0}\right]\right] \\
& =L_{x}^{-1}\left[\frac { 1 } { s ^ { 2 } } L _ { x } \left[\left(1-\pi^{2}\right) e^{\left(1-\pi^{2}\right) t}\right.\right. \\
& \left.\left.-e^{\left(1-\pi^{2}\right) t}\right]\right]=-\pi^{2} e^{\left(1-\pi^{2}\right) t} \frac{x^{2}}{2 !} \\
& =-\frac{(\pi x)^{2}}{2 !} e^{\left(1-\pi^{2}\right) t} \\
& u_{2}(x, t)=L_{x}^{-1}\left[\frac{1}{s^{2}} L_{x}\left[u_{1 t}+A_{1}\right]\right]=\pi^{4} e^{\left(1-\pi^{2}\right) t} \frac{x^{4}}{4 !} \\
& =\frac{(\pi x)^{4}}{4 !} e^{\left(1-\pi^{2}\right) t}
\end{aligned}
$$

and so on. In this manner the rest of the component of the series (2.6) have been calculated.

From the decomposition series (2.6), we again obtain the exact solution

$$
\begin{aligned}
u(x, t)=e^{\left(1-\pi^{2}\right) t} & -\frac{(\pi x)^{2}}{2 !} e^{\left(1-\pi^{2}\right) t}+\frac{(\pi x)^{4}}{4 !} e^{\left(1-\pi^{2}\right) t} \\
- & \frac{(\pi x)^{6}}{6 !} e^{\left(1-\pi^{2}\right) t}+\cdots \ldots \ldots \ldots \cdots \cdots
\end{aligned}
$$




$$
u(x, t)=e^{\left(1-\pi^{2}\right) t} \cos (\pi x)
$$

Example 2: Consider the nonlinear heat equation (2.2) with $\in=-2$ and $m=3$, that is,

$$
u_{t}=u_{x x}-2 u^{2}
$$

with initial condition $u(x, 0)=\frac{1+2 x}{x^{2}+x+1}$ and the

boundary conditions $u(0, t)=\frac{1}{6 t+1}, u_{x}(0, t)=\frac{12 t+1}{(6 t+1)^{2}}$

For the solution of this equation in the $t$ direction, we use the recursive relation given by

(2.10), we get

$$
\begin{aligned}
& u_{0}(x, t)=\frac{1+2 x}{x^{2}+x+1} \\
& u_{1}(x, t)=L_{t}^{-1}\left[\frac{1}{s} L_{t}\left[u_{0 x x}+A_{0}\right]\right] \\
&=L_{t}^{-1}\left[\frac{1}{s} L_{t}[1]\left[u_{0 x x}+2 u_{0}^{3}\right]\right] \\
&=-\frac{6(1+2 x) t}{\left(x^{2}+x+1\right)^{2}}
\end{aligned}
$$

$u_{2}(x, t)=L_{t}^{-1}\left[\frac{1}{s} L_{t}\left[u_{1 x x}+A_{1}\right]\right]=\frac{36(1+2 x) t}{\left(x^{2}+x+1\right)^{3}}$

$u_{3}(x, t)=L_{t}^{-1}\left[\frac{1}{s} L_{t}\left[u_{2 x x}+A_{2}\right]\right]=-\frac{216(1+2 x) t^{3}}{\left(x^{2}+x+1\right)^{4}}$

and so on, in this manner the rest of the terms of decomposition series have been calculated by using Mathcad7. Substituting values of $\mathrm{u}_{0}, \mathrm{u}_{1}, \mathrm{u}_{2}, \mathrm{u}_{3} \ldots \ldots \ldots$ in the equation (2.6), we get the exact solution of equation (3.15) in $t$ direction.

$$
\begin{gathered}
u(x, t)=\frac{1+2 x}{x^{2}+x}+\frac{6(1+2 x) t}{\left(x^{2}+x+1\right)^{2}}+\frac{36(1+2 x) t}{\left(x^{2}+x+1\right)^{3}} \\
-\frac{216(1+2 x) t^{3}}{\left(x^{2}+x+1\right)^{4}}
\end{gathered}
$$

This result can be verified through substitution. On the other hand, to obtain the solution in the $\mathrm{x}$ direction, we proceed as before to determine the individual term of the series (2.6)

$u_{0}(x, t)=u(0, t)+x u_{0}(0, t)=\frac{1}{6 t+1}+x \frac{12 t+1}{(6 t+1)^{2}}$

$$
\begin{aligned}
& u_{1}(x, t)=L_{x}^{-1}\left[\frac{1}{s^{2}} L_{x}\left[u_{0 t}+A_{0}\right]\right]=L_{x}^{-1}\left[\frac{1}{s^{2}} L_{x}\left[u_{0 t}+2 u_{0}^{3}\right]\right] \\
& =-x^{2} \frac{(18 t+2)}{(6 t+1)^{3}}+x^{3} \frac{\left(-72 t^{2}+1\right)}{(6 t+1)^{4}}+x^{4} \frac{\left(180 t^{2}+30 t+1\right)}{(6 t+1)^{5}}+ \\
& x^{5} \frac{\left(432 t^{3}-108 t^{2}-36 t-2\right)}{(6 t+1)^{6}}+\cdots \ldots .
\end{aligned}
$$

and so on. In this manner the rest terms of the decomposition series (2.6) have been calculated. Substituting values of $\mathrm{u}_{0}, \mathrm{u}_{1}, \mathrm{u}_{2}, \mathrm{u}_{3} \ldots \ldots \ldots \ldots$ in the equation (2.6), we get again exact solution of equation (3.15) in $x$ direction.

$$
\begin{aligned}
u(x, t)=\frac{1}{6 t+1} & +x \frac{12 t+1}{(6 t+1)^{2}}-x^{2} \frac{(18 t+2)}{(6 t+1)^{3}} \\
& +x^{3} \frac{\left(-72 t^{2}+1\right)}{(6 t+1)^{4}} \\
& +x^{4} \frac{\left(180 t^{2}+30 t+1\right)}{(6 t+1)^{5}} \\
& +x^{5} \frac{\left(432 t^{3}-108 t^{2}-36 t-2\right)}{(6 t+1)^{6}} \\
& +\cdots \ldots \ldots \ldots
\end{aligned}
$$

This result can be verified through substitution.

\section{CONCLUSION}

In this article, Laplace decomposition method is applied to solve linear and nonlinear heat equation in $t$ direction as well as $\mathrm{x}$ direction by taking the Laplace transform wit respect to $\mathrm{t}$ as well as $\mathrm{x}$ respectively. In both directions we get the same exact or approxi- mate solution. The results of two examples are comparing with ADM. The result of these two examples tell us that both methods can be used alternatively for the equation (1.1).

\section{REFERENCES}

[1] G. Adomian, Solving Frontier Problems of Physics: The Decomposition Method, Kluwer, Dordrecht, 1994.

[2] G. Adomian, Serrano SE, J. Appl. Math. Lett., vol. 2, pp. 161, 1995.

[3] G. Adomian, Nonlinear Stochastic Operator Equations, Academic Press, Orlando, 1986.

[4] G. Adomian, Nonlinear Stochastic Systems Theory and Applications to Physics, Kluwer Academic Publishers, Dordrecht, 1989.

[5] E. Zauderer, Partial Differential Equations of Applied Mathematics, Wiley, New York, 1983.

[6] B. A.-Hamid, "Exact Solutions of some Nonlinear Evolution Equations Using Symbolic Computations," Computers \& Mathematics with Applications, vol. 40, no. 291302, 2000. 